\title{
CERVICOTHORACIC TUMORS: RESULTS OF RESECTION BY THE "HEMI-CLAMSHELL" APPROACH
}

Robert J. Korst, MD

Michael E. Burt, MD, PhD

\begin{abstract}
Objectives: Our goal was to describe the "hemi-clamshell" approach for the resection of primary and metastatic tumors of the cervicothoracic junction, evaluate its morbidity and mortality, and present survival data on a series of 42 patients who underwent resection with the use of this technique. Methods: We conducted a retrospective review of the records of all patients of a single surgeon undergoing resection of tumors of the cervicothoracic junction. Data collected includes tumor type and involvement, type of resection, complications, and survival. Results: Forty-two patients underwent resection of various primary $(n=28)$ and metastatic $(n=14)$ tumors of the cervicothoracic junction over 6.5 years by means of the hemiclamshell approach. En bloc resection of the tumor and invaded structures was successful in all but two patients (5\%), who required an additional posterolateral thoracotomy to facilitate removal of tumor invading the posterior chest wall. Invaded structures that were resected included lung $(n=22)$, vertebral body $(n=7)$, chest wall $(n=8)$, central veins $(n=10)$, thyroid $(n=3)$, carotid artery $(n=1)$, and cervical esophagus $(n=1)$. Four major complications occurred in three patients, and nine minor complications occurred in eight patients. There were no deaths. The overall 5-year actuarial survival was $67.4 \%$. Conclusions: Tumors of the cervicothoracic junction are represented by a variety of histologic types and can be both primary and metastatic. The hemi-clamshell approach is a successful technique for the exposure and resection of these tumors. This approach has significant advantages over other previously reported techniques. The complication rate is low and the mortality rate is zero in this series, the largest yet reported. Long-term survival is acceptable if complete resection can be performed. (J Thorac Cardiovasc Surg 1998;115:286-95)
\end{abstract}

$T^{\mathrm{h}}$ he cervicothoracic junction is an anatomically complex region that has traditionally posed a problem for surgical access. Important neurovascular structures traverse this area, making exposure difficult. Tumors arising in the cervicothoracic junction compound this problem because they distort normal anatomy, invade local structures, and may be

From the Thoracic Service, The Memorial Sloan-Kettering Cancer Center, New York, N.Y.

Read at the Twenty-third Annual Meeting of The Western Thoracic Surgical Association, Napa, Calif., June 25-28, 1997.

Received for publication July 8, 1997; revisions requested July 28, 1997; revisions received Sept. 23, 1997; accepted for publication Sept. 23, 1997.

Address for reprints: Michael E. Burt, MD, PhD, Thoracic Service, The Memorial Sloan-Kettering Cancer Center, 1275 York Ave., New York, NY 10021.

Copyright (C) 1998 by Mosby, Inc.

$0022-5223 / 98 \$ 5.00+0 \quad \mathbf{1 2 / 6 / 8 6 3 3 1}$ associated with varying degrees of fibrosis, making identification of important structures difficult.

Malignant tumors encountered in this region, whether primary or metastatic, represent a heterogeneous group of tumors that tend to be locally aggressive and frequently recur after surgical resection. ${ }^{1-5}$ The risk of injury to important neurovascular structures has made the prospect of radical resection formidable, even though complete resection offers the best hope for cure. ${ }^{6}$ Multiple techniques have been described to provide access to this region. However, because no technique is without its drawbacks, the debate regarding the optimal approach continues.

The purpose of this report is threefold. First, we describe the "hemi-clamshell" approach for the resection of both primary and metastatic tumors of the cervicothoracic junction. This approach, consisting of a partial median sternotomy combined with 


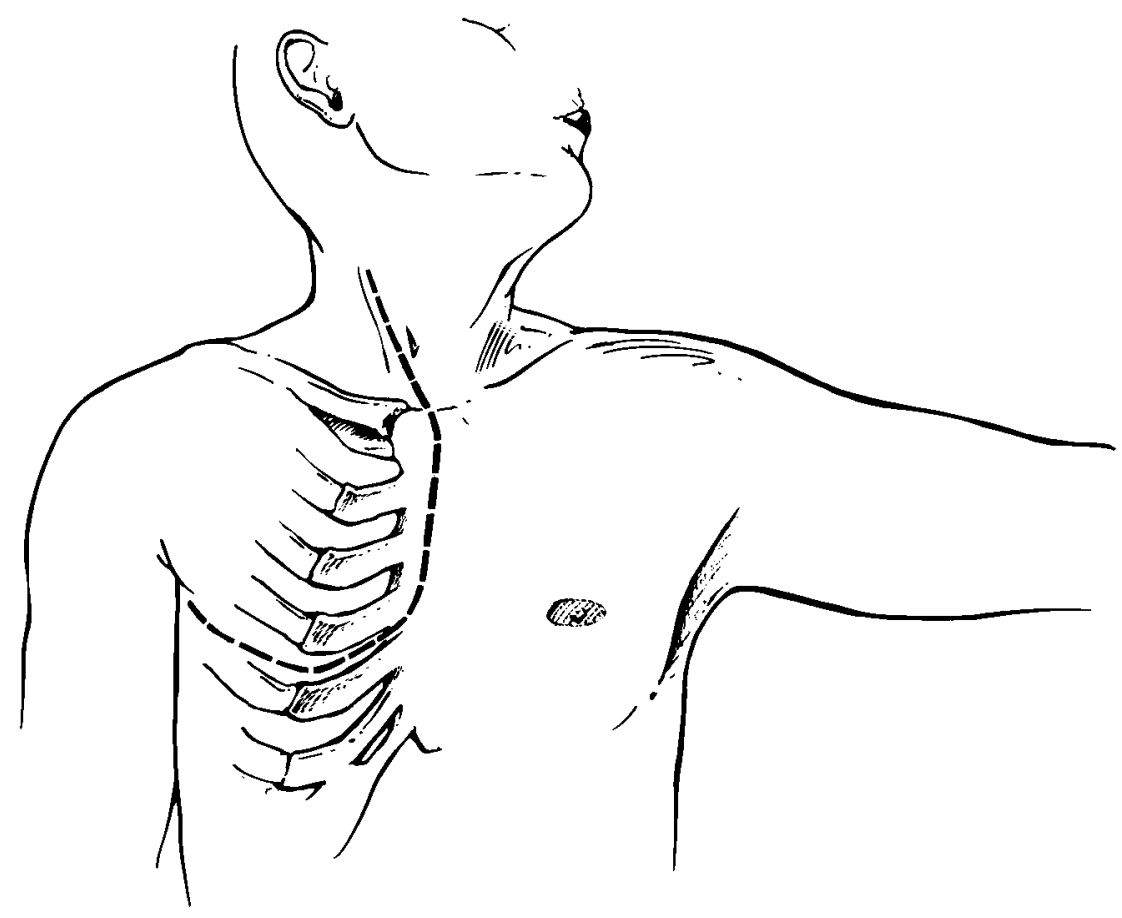

Fig. 1. Optimal patient positioning to perform a right-sided hemi-clamshell approach for the resection of tumors in the cervicothoracic junction. The broken line indicates where the skin incision should be placed. See text for description.

an anterior thoracotomy and neck incision, provides exceptional exposure for the resection of tumors in this area, as well as for resection of associated structures such as the lung and vertebral body. Second, the morbidity and mortality of this technique are evaluated. Third, survival data are presented for a series of 42 patients with both primary and metastatic tumors of the cervicothoracic junction who underwent the hemi-clamshell approach for the resection of their tumors.

\section{Methods}

A review of a prospective computerized database of a single surgeon (M.E.B.) identified 42 patients who underwent resection of primary and metastatic tumors of the cervicothoracic junction with the use of the hemi-clamshell approach. Data collected included tumor type, locoregional extent, type of resection, complications, and survival. Actuarial survival was computed by the method of Kaplan and Meier ${ }^{7}$ using the statistical software package SPSS Advanced Statistics 7.5 (SPSS Inc., Chicago, Ill.).

The hemi-clamshell approach was performed as follows. The patient is placed supine and undergoes general anesthesia. After intubation with a double-lumen endobronchial tube, the ipsilateral upper extremity is tucked at the side to facilitate manipulation of the chest wall without fracturing the clavicle. The contralateral upper extremity is abducted and the neck is extended with the head turned to the contralateral side. The patient is then prepared and draped in sterile fashion from the chin to the umbilicus. Fig. 1 demonstrates the optimal position of the patient.

An anterolateral thoracotomy incision is made from the sternum to the anterior axillary line. Electrocautery is used to divide the soft tissue of the chest wall, and the fourth intercostal space is entered. Care is taken to divide the pectoralis major proximal to its insertion onto the ribs to allow reapproximation without tearing of this muscle. The ipsilateral lung is collapsed and the pleural cavity is explored for findings that would preclude resection (diffuse pleural metastases). Once the decision to proceed has been made, the intercostal space is opened out to the midclavicular line and the skin incision is continued cephalad up the middle of the sternum and the anterior border of the ipsilateral sternocleidomastoid muscle. Fig. 1 depicts the skin incision in its entirety.

A partial sternotomy is made in the midline from the jugular notch down to the fourth intercostal space and then carried out into this intercostal space. The internal thoracic vessels are ligated and a sternal retractor is placed to elevate the chest wall and clavicle, exposing the mediastinum and ipsilateral hemithorax. Dissection is continued in the neck by dividing the platysma and strap muscles, exposing the carotid sheath and the thyroid gland. A self-retaining retractor is placed into the neck and the thyroid is retracted medially, exposing the cervical esophagus and spine. The carotid sheath structures are 

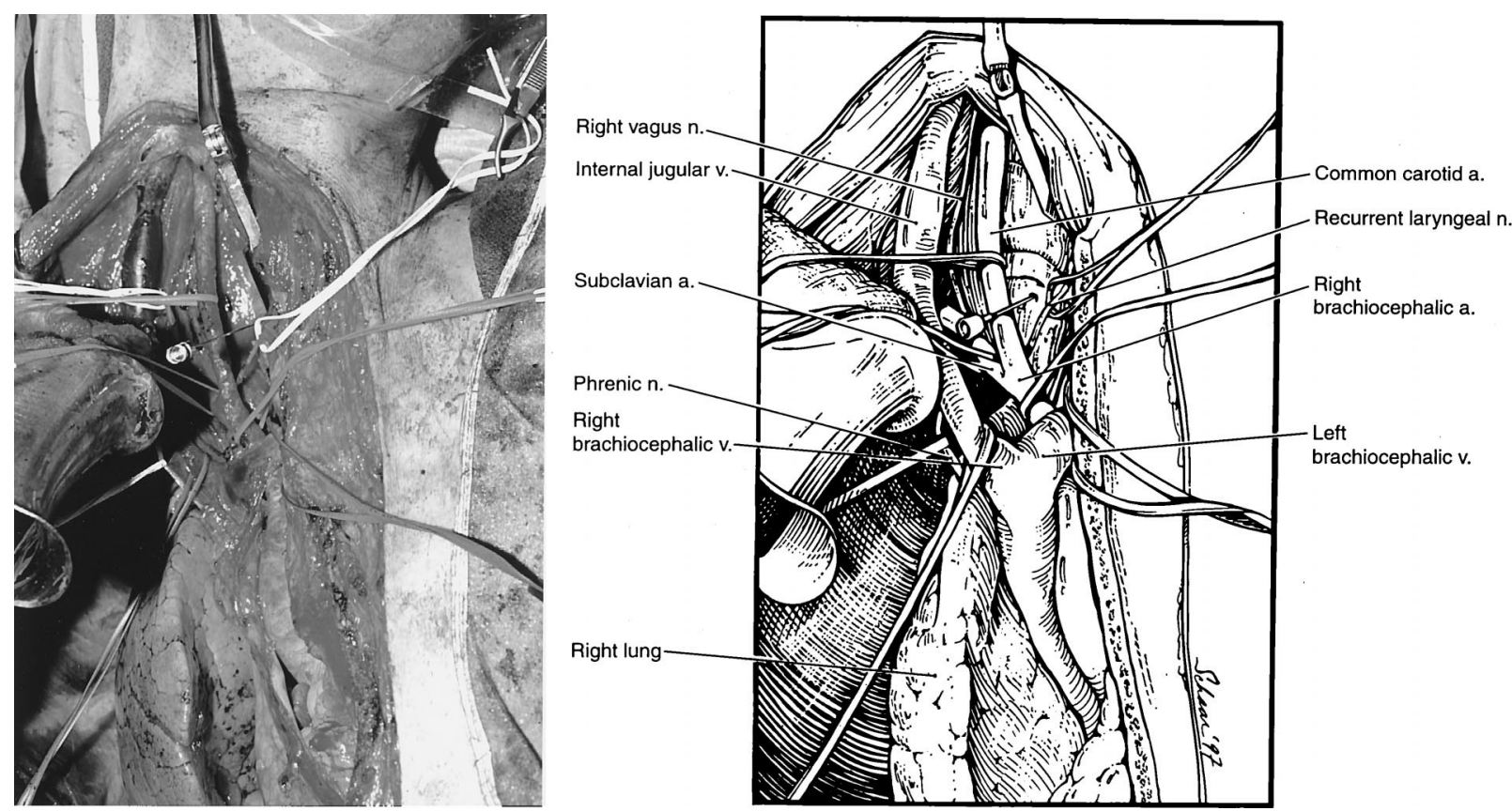

Fig. 2. Operative photograph (left) and corresponding diagram (right) demonstrating the exposure obtained by means of the hemi-clamshell approach. The view is mainly anterior, but slightly oblique from the right. The retractor is reflecting the right chest wall superiorly and laterally. Important structures are easily dissected and seen in their entirety in the neck and the chest. Note the excellent visualization of the right pulmonary hilum and cervicothoracic vertebral bodies. The needle is placed in the T2 vertebral body. $a$. ., Artery; $v$., vein; $n$., nerve.

well exposed, dissected individually, and easily followed from the neck down into the mediastinum. The recurrent laryngeal and phrenic nerves are also easily dissected and visualized throughout their entire course in the neck and thorax. Fig. 2 demonstrates the hemi-clamshell approach in its entirety, and Fig. 3 reveals the cervical portion of the dissection in greater detail.

The tumor present in the cervicothoracic junction can now be readily dissected free from the surrounding neurovascular structures. En bloc resection of neighboring involved structures such as the vertebral bodies, ribs, veins, arteries, nerves, and thyroid is now possible because of this wide exposure. The pulmonary hilum is also easily dissected, allowing for various anatomic lung resections to be performed without the need for a separate thoracotomy incision.

After resection of the tumor, chest tubes are placed, the sternum is reapproximated with wires, and pericostal sutures are placed to close the intercostal space. The remainder of the closure is performed in standard fashion.

\section{Results}

A total of 42 patients underwent resection of cervicothoracic tumors via the hemi-clamshell approach between March 1990 and October 1996. The same surgeon performed all procedures. There were
21 female and 21 male patients whose ages ranged from 13 to 75 years (median 42 years). Twenty-eight lesions were primary (67\%) and $14(33 \%)$ were metastatic. Presenting symptoms are detailed in Table I. In the majority of the patients who were free of symptoms, the lesions were detected incidentally as part of a metastatic work-up. Table II displays the various histologic types encountered and their relative frequencies. The hemi-clamshell approach was performed on the left in 22 patients, on the right in 17, and bilaterally in 3. Figs. 4 and 5 display representative computed tomographic scans of tumors resected via the hemi-clamshell approach.

Therapies instituted before resection included external beam radiation therapy in five, chemotherapy in 20 , and radioactive iodine in two. Three patients had resection attempted elsewhere in the recent past, and 13 patients with metastatic disease had previous resection of their primary tumors. Adjuvant therapy included external beam radiotherapy in 19, chemotherapy in three, intraoperative placement of iodine 125 brachytherapy im- 

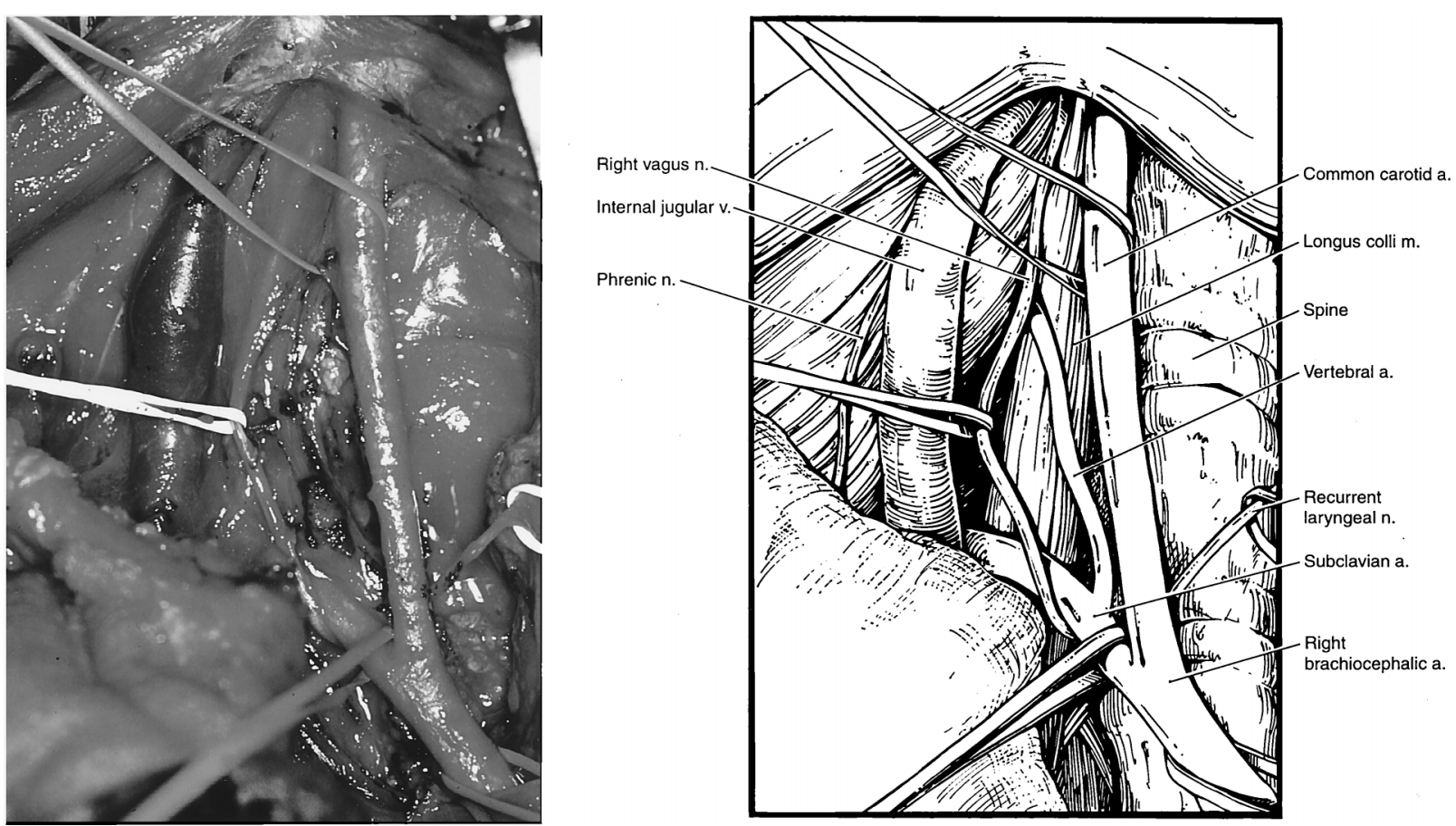

Fig. 3. Operative photograph (left) and corresponding diagram (right) of the cervical portion of the dissection. The phrenic nerve is seen overlying the anterior scalene muscle. If necessary, this muscle is divided later in the dissection to expose the brachial plexus. $a$., Artery; $v$., vein; $n$., nerve; $m$., muscle.

Table I. Presenting symptoms of patients with tumors of the cervicothoracic junction

\begin{tabular}{lc}
\hline \multicolumn{1}{c}{ Symptom } & No. of patients \\
\hline Pain (chest wall, neck, shoulder, & 16 \\
$\quad$ arm) & \\
Arm parasthesias & 6 \\
Neck swelling & 3 \\
Dysphagia & 2 \\
Dyspnea & 2 \\
Arm weakness & 2 \\
Spinal cord compression & 2 \\
Neck mass & 2 \\
Cough & 1 \\
Venous thrombosis (internal jugular) & 1 \\
Asymptomatic & 8 \\
\hline
\end{tabular}

plants in four, systemic radioactive iodine in two, and immunotherapy in one.

En bloc resection of the tumor with surrounding invaded structures was readily achieved through the hemi-clamshell approach in all but two patients. A separate posterolateral thoracotomy was required in these two patients $(5 \%)$ to facilitate removal of tumor invading the posterior chest wall. All gross tumor was resected in 35 patients $(83 \%)$; four patients had a microscopi-
Table II. Tumor types encountered at the cervicothoracic junction

\begin{tabular}{lc}
\hline \multicolumn{1}{c}{ Tumor type } & No. of patients \\
\hline Primary & 28 \\
Bronchogenic carcinoma & 12 \\
Sarcoma & 10 \\
Thyroid carcinoma & 3 \\
Thymic & 2 \\
Lymphoma & 1 \\
Metastatic & 14 \\
Germ cell/teratoma & 6 \\
Sarcoma & 4 \\
Melanoma & 1 \\
Thyroid carcinoma & 1 \\
Breast carcinoma & 1 \\
Renal cell carcinoma & 1 \\
\hline
\end{tabular}

cally positive margin. Table III lists the structures and organs involved by tumor that required resection along with the mass. No structures were inadvertently sacrificed.

A total of four major complications occurred in three patients $(9.5 \%)$. Two of these patients underwent a posterolateral thoracotomy, as well as the hemi-clamshell approach. Fungal sepsis and subsequent renal failure occurred in one patient, another 


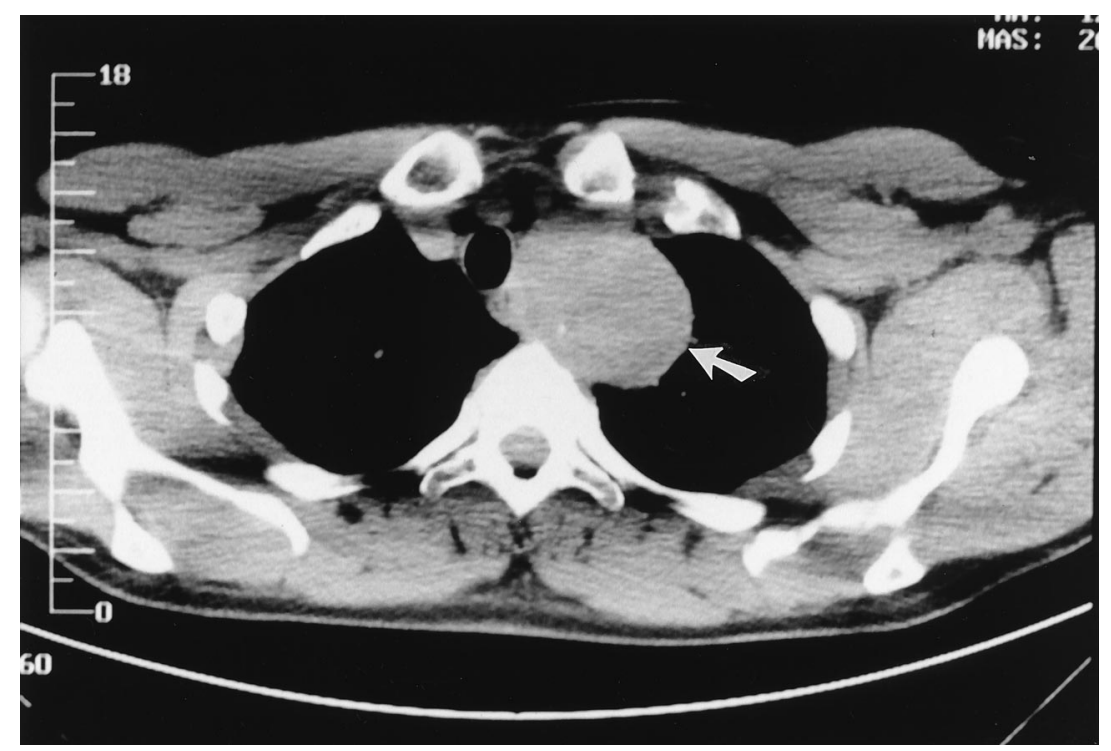

Fig. 4. Computed tomographic scan at the T1 level of a 39-year-old man who had left shoulder and arm pain. The tumor is indicated with an arrow. A complete resection was obtained through the left hemi-clamshell approach and pathologic examination revealed a chordoma of the mediastinum.

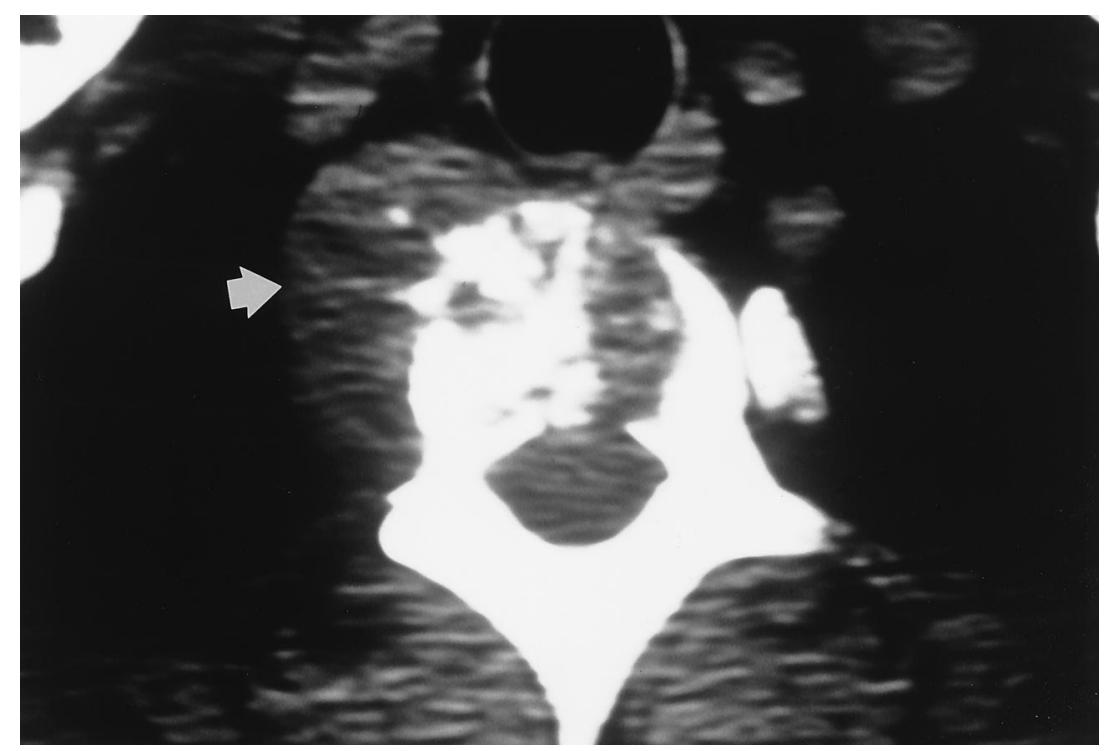

Fig. 5. Computed tomographic image at the $\mathrm{T} 3$ level of a 33 -year-old man with a history of a surgically treated lower extremity soft tissue sarcoma. The tumor is indicated with an arrow. Obvious destruction of the T3 vertebral body is noted. Pathologic examination revealed metastatic leiomyosarcoma.

had postoperative streptococcal pneumonia, and a third had a chylothorax that responded to conservative management. Nine minor complications occurred in eight patients (21.4\%), and these are listed in Table IV. There were no deaths. In addition, no patients had any long-term neurologic sequelae or deformities attributable to the operative approach.
The overall actuarial 5-year survival was $67.4 \%$ and the median survival has not yet been reached. Fig. 6A displays overall actuarial survival for all 42 patients, and Fig. 6B demonstrates actuarial survival according to whether the tumor was primary or metastatic. Twenty-five patients $(60 \%)$ are alive without disease, six (14\%) are alive with active 
Table III. Associated structures resected with cervicothoracic tumors

\begin{tabular}{lc}
\hline Organ/tissue resected & No. of patients \\
\hline Lung & 22 \\
Pneumonectomy & 3 \\
Bilobectomy & 1 \\
Lobectomy & 3 \\
Segmentectomy & 1 \\
Wedge resection & 14 \\
Vertebral body & 7 \\
Chest wall & 8 \\
Central veins & 10 \\
Phrenic nerve & 5 \\
Thyroid & 3 \\
Carotid artery & 1 \\
Cervical esophagus & 1 \\
\hline
\end{tabular}

disease, ten $(24 \%)$ died as a result of their tumors, and one $(2 \%)$ died of an unrelated cause.

\section{Discussion}

Exposure for the resection of tumors involving the cervicothoracic junction is controversial and has been the subject of multiple previous reports, the majority of which exist in the neurosurgical and orthopedic literature and describe anterior, lateral, and posterior approaches for the exposure of the cervicothoracic spine. ${ }^{8-11}$ However, no single technique is without drawbacks and therefore no agreement exists as to what constitutes the optimal approach.

The posterior approach to bronchogenic carcinomas in the superior sulcus as described by Paulson ${ }^{12}$ provides adequate exposure for resection of apical lung tumors invading the posterior aspect of the ribs and cervicothoracic junction but makes resection of anterior apical tumors difficult, especially in the presence of extensive extrathoracic invasion. Access to the pulmonary hilum is excellent, facilitating any type of pulmonary resection that may be necessary. Surgical access for the resection of bronchogenic carcinoma invading the thoracic inlet has also been described by Dartevelle and associates ${ }^{13}$ through an L-shaped transcervical incision. With this approach, however, control of the pulmonary vessels at the hilum and chest wall resection below the second rib are difficult, necessitating a separate posterolateral thoracotomy in 20 of 29 patients $(69 \%)$ in this series. In addition, this approach involves the resection of the medial half of the clavicle, which contributes to shoulder instability, weakness, and deformity. ${ }^{14,15}$ Access for complete mediastinal lymph node dissec-
Table IV. Minor complications after cervicothoracic tumor resection via hemi-clamshell approach

\begin{tabular}{lc}
\hline \multicolumn{1}{c}{ Complication } & No. of occurrences \\
\hline Upper extremity edema* & 3 \\
RLN injury $\dagger$ & 1 \\
Neck seroma & 1 \\
Neck hematoma & 1 \\
Superficial wound infection & 1 \\
Atrial fibrillation & 1 \\
$\quad$ Urinary tract infection & 1 \\
*Self limited. & \\
$\dagger$ Recurrent laryngeal nerve traction injury. Spontaneous recovery of \\
function occurred.
\end{tabular}

tion, a necessary component of many oncologic operations, is also very limited.

Macchiarini and coworkers ${ }^{6}$ described an identical technique for the resection of both primary and metastatic nonbronchogenic tumors of the thoracic outlet in 14 patients. Four of these patients again required the performance of additional incisions to facilitate exposure for tumor resection, and the medial half of the clavicle was resected in all patients. One patient died after the operation and there was one late death resulting from progressive disease, but the remaining 12 patients were without evidence of disease at a median follow-up of 3.4 years.

Nazari ${ }^{16}$ recently reported a modification of the anterior transcervical approach (Dartevelle technique) in which the clavicle is not divided. Although shoulder stability may be improved by wiring the clavicle back to the manubrium, this approach is still hampered by the exposure problems characteristic of the Dartevelle technique.

Grunenwald and Spaggiari ${ }^{14}$ agree with Nazari concerning the disadvantages of resecting the clavicle when resecting tumors of the cervicothoracic junction. These authors describe an anterior approach similar to that described by Nazari except that the sternoclavicular joint is maintained and the manubrium is divided to "open" the access to the thoracic inlet. The advantage of this technique is improved shoulder stability attained by leaving the sternoclavicular joint and the muscular attachments of the clavicle intact. However, in their series of eight patients, four required an additional posterolateral thoracotomy to facilitate the performance of pneumonectomy and vertebrectomy.

We have previously reported the hemi-clamshell technique for the exposure and resection of spinal tumors from the C4-T3 levels, as well as for 
A Overall
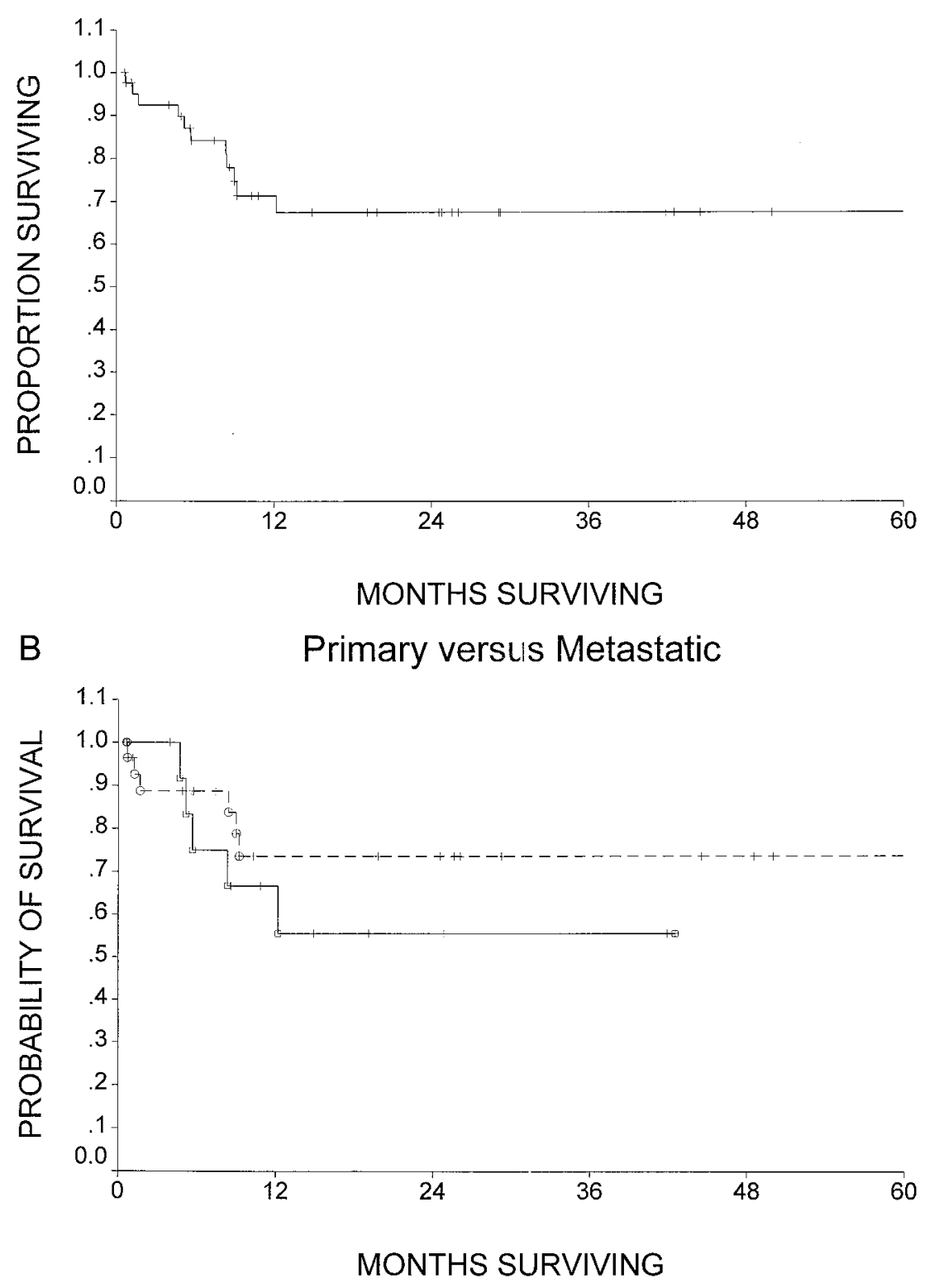

Fig. 6. A, Actuarial survival curve for all 42 patients who underwent resection by means of the hemi-clamshell approach. The 5-year actuarial survival is $67.4 \%$. B, Actuarial survival curves for 28 patients with primary tumors (broken line) and 14 patients with metastatic tumors (solid line) of the cervicothoracic junction who underwent resection by means of the hemi-clamshell approach. The 5-year actuarial survival for the patients with primary tumors is $73.6 \%$, and the 3 -year actuarial survival for the patients with metastatic tumors is $55.6 \%$. Five-year survival was not able to be calculated for those with metastatic lesions because of insufficient long-term follow-up.

a small series of soft tissue tumors at the cervicothoracic junction. ${ }^{17,18}$ A similar technique has also been described for the resection of both mediastinal tumors with extensive hemithoracic involvement ${ }^{19}$ and superior sulcus lung tumors. ${ }^{20}$
The present report represents the largest series ever reported for the resection of tumors in this area by any approach. The hemi-clamshell technique has several advantages over the approaches previously described by other authors. First, ex- 
posure of the pulmonary vessels at the hilum is excellent, facilitating the performance of major anatomic pulmonary resections, which were required in $19 \%$ of the patients in this series, including three pneumonectomies. Second, there is no limit on the size of the chest wall resection that can be performed by means of the hemiclamshell approach. Third, the clavicle is not divided and the sternoclavicular joint is left intact, which diminishes postoperative discomfort and improves shoulder stability and appearance. Fourth, the hemi-clamshell approach is far better than any other described technique for the performance of mediastinal lymph node dissection, a necessary part of many tumor resections.

The only disadvantage of the hemi-clamshell approach in the resection of tumors of the cervicothoracic junction is the exposure of the posterior chest wall and neural foramina. This problem is shared by the other anterior approaches to these tumors. As a result, we continue to use the traditional posterior approach described by Paulson ${ }^{12}$ to remove superior sulcus tumors invading the posterior aspect of the first rib, reserving the hemi-clamshell technique for anterolateral tumors. In the uncommon instance in which a tumor involves the posterior apical chest wall and invades the cervicothoracic junction structures anteriorly as well, two separate incisions may be necessary. We found this to be the case in two $(5 \%)$ of our 42 patients.

The hemi-clamshell approach has been criticized by some because of questionable ability to perform vertebral body resections and because of the possibility of postoperative flail chest. ${ }^{14,20}$ In our series of 42 patients, we successfully performed seven vertebral body resections and the flail chest phenomenon was not seen in any patients. Figs. 2 and 3 demonstrate the excellent access to the cervicothoracic vertebral bodies.

In summary, we describe the hemi-clamshell technique for the resection of primary and metastatic tumors involving the cervicothoracic junction and report survival data on the largest series of patients treated for tumors in this area. As described, this technique has significant advantages over approaches previously published in the thoracic surgical literature. The complication rate is low and the mortality rate is zero in this series of 42 patients. Long-term survival is encouraging provided that a complete resection can be performed.

\section{REFERENCES}

1. Witkin GB, Miettinen M, Rosai J. A biphasic tumor of the mediastinum with features of synovial sarcoma. Am J Surg Pathol 1989;13:490-9.

2. Venuta F, Pescarmona EO, Rendina EA, Ciriaco P, De Giacomo T, Ricci C. Primary osteogenic sarcoma of the mediastinum. Scand J Thorac Cardiovasc Surg 1993;27:16973.

3. Simon D, Goretzki PE, Witte J, Roher HD. Incidence of regional recurrence guiding radicality in differentiated thyroid carcinoma. World J Surg 1996;20:860-6.

4. Anderson TM, Moy PM, Holmes EC. Factors affecting survival in superior sulcus tumors. J Clin Oncol 1986;4:1598603.

5. King RM, Pairolero PC, Trastek VF, et al. Primary chest wall tumors: factors affecting survival. Ann Thorac Surg 1986;41: 597-601.

6. Macchiarini P, Dartevelle P, Chapelier A, Lenot B, Cerrina J, Ladurie FLR, et al. Technique for resecting primary and metastatic tumors of the thoracic outlet. Ann Thorac Surg 1993;55:611-8.

7. Kaplan EL, Meier P. Nonparametric estimation from incomplete observations. J Am Stat Assoc 1958;53:457-81.

8. Hodgson AR, Stock FE, Fang HSY, Ong GB. Anterior spinal fusion: the operative approach and pathologic findings in 412 patients with Pott's disease of the spine. Br J Surg 1960;48: $172-8$.

9. Sundaresan N, DiGiacinto GV. Surgical approaches to the cervico-thoracic junction. In: Sundaresan N, Schnidek HH, Schiller AL, Rosenthal DI, editors. Tumors of the spine: diagnosis and clinical management. Philadelphia: WB Saunders; 1990. p. 358-68.

10. Kostuik JP. Surgical approaches to the thoracic and thoracolumbar spine. In: Frymoyer JW, editor. The adult spine: principles and practice. New York: Raven; 1991. p. 1243-66.

11. Benzel EC. The lateral extracavitary approach to the spine using the three-quarter prone position. J Neurosurg 1989;71: $837-41$.

12. Paulson DL. Carcinomas in the superior pulmonary sulcus. J Thorac Cardiovasc Surg 1975;70:1095-104.

13. Dartevelle P, Chapelier AR, Macchiarini P, Lenot B, Cerrina J, Ladurie FLR, et al. Anterior transcervical-thoracic approach for radical resection of lung tumors invading the thoracic inlet. J Thorac Cardiovasc Surg 1993;105:1025-34.

14. Grunenwald D, Spaggiari L. Transmanubrial osteomuscular sparing approach for apical chest tumors. Ann Thorac Surg 1997;63:563-6.

15. Jaklitsch MT, Rego A. Endorsement for sparing the clavicle in the transcervical approach to the thoracic inlet. $\mathrm{J}$ Thorac Cardiovasc Surg 1997;113:958-9.

16. Nazari S. Transcervical approach (Dartevelle technique) for resection of lung tumors invading the thoracic inlet, sparing the clavicle. J Thorac Cardiovasc Surg 1996;112:558-9.

17. Nazarro JM, Arbit E, Burt M. "Trap door" exposure of the cervicothoracic junction: technical note. J Neurosurg 1994; 80:338-41

18. Kraus DH, Huo J, Burt M. Surgical access to tumors of the cervicothoracic junction. Head Neck 1995;17:131-6.

19. Bains MS, Ginsberg RJ, Jones WG. The clamshell incision: an improved approach to bilateral pulmonary and mediastinal tumor. Ann Thorac Surg 1994;58:30-3.

20. Masaoka A, Ito Y, Yasumitsu T. Anterior approach for 
tumor of the superior sulcus. J Thorac Cardiovasc Surg 1979;78:413-5.

\section{Discussion}

Dr. Walter B. Cannon (Palo Alto, Calif.). Cervicothoracic tumors are challenging problems for the surgeons, as evidenced by the numerous surgical approaches that have been described by many surgical subspecialties including neurosurgeons, head and neck surgeons, orthopedic surgeons, and, of course, thoracic surgeons. As the authors have stated, the potential for damage to the neurovascular structures in this region is very high. Of further concern is the fact that approximately one third of the tumors that arise in this area are in the area of radiation for tumors of the breast and for lymphomas. With a latent period of many years and with increasing numbers of patients having been cured of their original malignant diseases, these tumors are undoubtedly going to be more prevalent. We as thoracic surgeons are going to be called on to treat them. For these reasons, this paper has real significance. These patients potentially can be cured if the tumor can be wholly removed with clear margins at the initial operation. Although lesser operations have been reported and have apparently been successful, significant cosmetic and functional problems, especially with the removal of the clavicle, have also been reported. This hemi-clamshell approach would be particularly appealing to surgeons who are comfortable in performing thoracotomies as well as sternotomies. I would be uncomfortable wiring the clavicle to the sternum and expect some kind of healing to occur without long-term pain, but I would be very comfortable wiring the sternum together and expect to have good healing, a stable chest, and a good cosmetic result.

It is remarkable that no deaths occurred in this series of 42 patients. Although the neurovascular structures are at a major risk for this procedure, no neurovascular structures were apparently damaged. The overall actuarial 5-year survival was $67.4 \%$, but the 5-year actuarial survival for the primary tumors was a remarkable $73.6 \%$. These results are as good as anyone could ever hope for, and the authors should be congratulated.

I have a few questions. You have described the operation and your results but have not mentioned what criteria you use to determine that these tumors are resectable. Does this series reflect all patients that you see with cervicothoracic tumors, or is this a very selected group of patients? What do you do with patients with arm, shoulder, or back pain and probable brachial plexus involvement? Also, what do you do with the patient with probable mediastinal adenopathy? How do you handle the patient who probably has unresectable disease and therefore cannot be cured and who may have also been irradiated in the cervicothoracic area?

Dr. Korst. We use the preoperative computed tomographic scan to determine which tumors are resectable; however, in many cases, operative exploration is needed. The best patient for this approach is a patient who has a tumor that tends to be anterior on the computed tomographic scan. In the case of a superior sulcus tumor that appears to be invading the posterior aspect of the first rib, we will do the posterolateral approach as described by
Paulsen. However, if the tumor is more anterior and lateral, we will use the hemi-clamshell approach.

Dr. Cannon. One of the issues that arises is that these tumors are not encountered until there is pain, and there is often pain associated with involvement of the brachial plexus. If the brachial plexus were involved in some "minor" fashion, so that only a portion needed to be removed, understanding the fact that some arm paralysis would be associated with the procedure, would you do that?

Dr. Korst. We have taken the T1 nerve roots; we have not had patients in whom we have needed to take more of the brachial plexus.

Dr. Cannon. What do you do if the patient has obvious mediastinal lymphadenopathy?

Dr. Korst. All patients have a preoperative computed tomographic scan to evaluate the mediastinum. If bulky nodes are present, a tissue diagnosis is obtained. If diffuse mediastinal metastases are present, resection is usually not attempted.

Dr. Cannon. One of the issues that arises is the fact that some of these tumors are induced by radiation therapy and often develop in an irradiated area. What we often encounter is a patient who has a lot of pain in this area, has an obvious tumor, and probably has unresectable disease. How do you handle those problems? These are very difficult problems that are going to become increasingly prevalent, because a lot of these patients with breast cancer and lymphomas were cured of the initial disease, perhaps 20 years ago, and now have a major problem that we have to treat.

Dr. Korst. We do an exploratory operation if we think the disease is resectable. Two of the patients in this series actually had postradiation tumors, and both had good results after exploration and resection.

Dr. Cannon. One last question: Have you had to turn down a number of patients because of inoperability, large tumors, or extensive involvement of the brachial plexus?

Dr. Korst. I do not have that data.

Dr. Cannon. The point I am trying to make is that sometimes an arm has to be amputated because of the involvement of this area, and that is a real problem.

Dr. John R. Benfield (Sacramento, Calif.). I do not recall your mentioning the right recurrent laryngeal nerve. Did you ever have to sacrifice that as part of the planned part of the operation? Second, were there any instances in which that nerve was damaged when you had not intended it to be?

Dr. Korst. We did not have to intentionally sacrifice the recurrent laryngeal nerve. We did have one instance in which there was a traction injury to the nerve, because the vocal cord paralysis was self-limited.

Dr. Richard G. Fosburg (Del Mar, Calif.). My curiosity is piqued by the fact that a third of the patients in this series had what would be characterized as limited pulmonary resections, one segmentectomy and 14 wedge resections. Has there been a change in your philosophy about the treatment of malignant pulmonary disease at Memorial Sloan Kettering?

Dr. Korst. The anatomic pulmonary resections are basically performed for the primary lung cancers. If the 
tumor is not a primary lung cancer but invades the lung, a wedge resection is performed.

Dr. Fosburg. In your abstract you indicated that there are 28 primary tumors, 12 of which were lung tumors. Are you implying that the 12 patients who had lung tumors were not subjected to wedge resection?

Dr. Korst. The majority of the patients with lung cancers had anatomic resections. There were instances in which wedge resection or segmentectomy was performed.

Dr. James B. D. Mark (Stanford, Calif.). Just a technical question. All of us find ourselves having to extend incisions when we have started with one approach. Do you plan this particular incision right from the start, or have you done, perhaps, a sternotomy or a thoracotomy and then had to extend it into the other portion of the incision?

Dr. Korst. We plan it right from the start. However, we start the procedure with an anterior thoracotomy, because we want to eliminate the possibility of pleural disease that would make us have to stop the operation. After the thoracotomy, we go up the sternum and up the neck.

Dr. Mark. Do you think you could remove some of the anterior tumors with a standard sternotomy and not have to extend into the chest? You determine this on the computed tomographic scan, I presume.

Dr. Korst. Yes, the computed tomographic scan determines the approach. These tumors extend from the chest up into the thoracic inlet, and I think that area is hard to safely approach through just a sternotomy, even with a neck extension.

The exposure with this approach, being able to raise the chest wall up like a flap, is phenomenal. We have had no added morbidity from adding the thoracotomy to the sternotomy.

Dr. Mark. Can we call this maximally invasive surgery?

Dr. Korst. Yes.

Dr. Paul F. Waters (Los Angeles, Calif.). In the patients with lung cancer, do you evaluate the lymph nodes before taking them out, or do you do mediastinoscopy first?

Dr. Korst. The primary lung cancers we treat like superior sulcus tumors. Most of them are staged with cervical mediastinoscopy.

Dr. Waters. Do they get the standard preoperative therapy?

Dr. Korst. The decision to give preoperative radiotherapy is surgeon- and patient-specific.

\section{Availability of Journal back issues}

As a service to our subscribers, copies of back issues of The Journal of Thoracic and Cardiovascular Surgery for the preceding 5 years are maintained and are available for purchase from Mosby at a cost of $\$ 16.00$ per issue until inventory is depleted. The following quantity discounts are available: $25 \%$ off on quantities of 12 to 23, and one third off on quantities of 24 or more. Please write to Mosby, Inc., Subscription Services, 11830 Westline Industrial Drive, St. Louis MO 63146-3318, or call 800-453-4351 or 314-453-4351 for information on availability of particular issues. If unavailable from the publisher, photocopies of complete issues may be purchased from UMI, 300 N. Zeeb Rd., Ann Arbor, MI 48106, 313-761-4700. 